\title{
Comida, ética y tradiciones desde la frontera México-Estados Unidos
}

Tortilla Soup | María Ripoll | 2001

\section{María del Carmen Camacho Gómez" y Claudia Ivett Romero Delgado}

Universidad Panamericana, México

Recibido: 11 de enero 2020; aceptado: 30 de marzo 2020

\begin{abstract}
Resumen
El auge en la producción de películas y programas televisivos sobre comida en todo el mundo justifican su investigación, pues muestran la cultura y tradiciones en la sociedad. Cuando las personas dejan su país de origen para vivir en un nuevo lugar, crean costumbres que se mezclan con las que ya tenían. El proceso se ve reflejado en diferentes aspectos, como la comida, y un ejemplo de esta fusión cultural se muestra en la cinta Tortilla Soup, que habla de los cambios en la vida de los mexicanos que emigran a Estados Unidos para encontrar el tan deseado sueño americano.
\end{abstract}

Palabras Clave: Comida | Ética | Tradiciones | Familia | Cultura

Food, ethics and traditions from the Mexico - United States border

Abstract

The increase in the production of food films and television programs around the world justify its research as they show the culture and traditions of societies. When people leave their countries and move to a new place, they create customs that merge with the old ones. The process is shown in different aspects such as food, and a good example of this is presented in the film Tortilla Soup, because it presents the changes in the life of Mexicans, who emigrate to the United States to find the desired American dream.

Keywords: Food | Ethics | Traditions | Family | Culture

\section{Introducción}

La migración de mexicanos a Estados Unidos se ha dado desde hace mucho tiempo; las personas se ven en la necesidad de abandonar su país buscando una mejor vida para sus familias.

Los inmigrantes mexicanos continúan siendo, por mucho, el grupo hispano más grande en Estados Unidos. De cada 100 migrantes, 65 son mexicanos y en total son 36.634.000 (Noe-Bustamante, Alberti y Rodriguez-Gitler, 2019).

La migración de mexicanos ha estado por años impulsada por trabajadores sin estudios superiores que se desempeñan en su mayoría en los sectores agrícola y de la construcción, pero en las últimas décadas esto ha cambiado, pues los hijos de estos migrantes ya nacieron en EE.UU. y tienen más probabilidades de obtener educa- ción a nivel superior y por tanto, a tener mejor calidad de vida en todos los aspectos. Esto implica que los negocios dedican más recursos a desarrollar y vender productos a los latinos, que su poder adquisitivo y visibilidad vayan en aumento.

La presencia de trabajadores mexicanos en el mercado laboral de Estados Unidos se ha incrementado y diversificado significativamente en los últimos años. Algunas de las causas son la brecha salarial, la generación de empleos de calidad y bien remunerados, la demanda de mano de obra y el establecimiento de redes sociales (Piñeiro y Acosta, 2015).

Los estudios que abordan los procesos de migración de México a Estados Unidos se han realizado en su mayoría partiendo de la teoría de la asimilación, en sus diferentes modalidades (Pérez-Soria, 2017). Los inmigrantes comienzan a apropiarse de un conjunto de

* Corresponding author: mcamacho@up.edu.mx 
caracterísitcas importantes de la sociedad de Estados Unidos como la posibilidad de cambios culturales (Portes y Zhou, 1993), pertenencia de elementos socioculturales, movilidad de ascenso en la economía y cambios donde la cultura se mezcla y surge una nueva tradición (Alba y Nee, 2003).

Muchos de los mexicanos que migran a Estados Unidos trabajan en agricultura, pero otros entran al mercado laboral de la fabricación de alimentos, donde los latinos indocumentados son el 13\% y los latinos con documentos, el $17 \%$ (Radford y Noe-Bustamante, 2019). Esto habla de que muchos encuentran en la comida un cambio en sus vidas, y también una forma de llevarse sus tradiciones.

Debemos tener en cuenta que la comida mexicana se prepara desde mucho tiempo antes que el río Bravo sirviera para delimitar una frontera; los migrantes se llevan las recetas de sus abuelas y madres para sentirse más cerca de sus raíces y de sus familias.

\section{Metodología}

Este trabajo tiene como objetivo abordar el tema de la migración México-Estados Unidos a partir del análisis de la película Tortilla Soup o Sopa de Tortilla (María Ripoll, 2001), que es la adaptación estadounidense de la película oriental Comer, beber y amar (Ang Lee, 1994).

Los ejes a considerar son la comida, la cultura y la ética. A partir del resumen de la película, se determinó que los momentos clave serían las cenas dominicales, en las que la familia Naranjo se reunía sin falta para hablar sobre lo más importante en la vida de cada uno.

El objeto de estudio de esta investigación es analizar si la migración debilita las tradiciones familiares y culturales de los que emigran a Estados Unidos, como es el caso de los Naranjo. Por un lado, el análisis se dará a través de la comida, pues para la sociedad en general, es el momento en que se reúnen y comparten con sus familias lo que viven día a día. Por otro lado, se revisará la identidad representada en las hijas Naranjo, que no se consideran mexicanas, pero tampoco del todo estadounidenses, pues comparten las dos culturas y esto genera un choque en ellas.

Asimismo se analizará la ética, las tradiciones y la comida, pues es el fundamento eminentemente social del ser humano y esto se da dentro del marco de las relaciones con los demás.

\section{Sinopsis: la comida reúne a las familias}

No se muestra en la cinta pero Martín Naranjo (Héctor Elizondo) y su esposa Ana, ambos mexicanos, emigran a California en donde Martín empieza a trabajar como cocinero al lado del que se convertirá en su mejor amigo, Gómez (Julio Oscar Mechoso), de origen cubano. Con el tiempo, Naranjo y su esposa forman una familia con tres hijas: Leticia (Elizabeth Peña), Carmen (Jacqueline Obradors) y Maribel (Tamara Mello). Lamentablemente, la esposa de Martín muere y el viudo se hace cargo de sus hijas al tiempo que busca mantener vivas sus raíces y su cultura latina.

La película inicia con imágenes de Martín preparando los platillos para la tradicional cena familiar de los domingos, a la que ninguna de sus hijas, ya adultas jóvenes, puede faltar. Cada domingo se sirven guisos tradicionales mexicanos y se discuten temas importantes para los Naranjo.

Mientras tanto, sus hijas organizan sus actividades para llegar a tiempo con su papá. Carmen es administradora y se da tiempo para una cita rápida con Jeff, su ex novio. Leticia es maestra en una escuela primaria, visita frecuentemente la iglesia y se encuentra con su amiga Yolanda. Maribel es la más pequeña y trabaja en una tienda de discos; conoce a Andy, un chico brasileño rechazado por una compañera de trabajo y sale con él.

Carmen y Leticia, ya en casa, se sientan a la mesa que está puesta como para una celebración. Leticia da gracias por los alimentos y mientras se sirven, platican en una mezcla de español e inglés que desagrada a Martín. Carmen anuncia que comprará un departamento, lo que molesta a su padre pues no lo consultó con él primero.

Yolanda llega con su hija April, a la que cuidarán mientras Yolanda va por su mamá al aeropuerto. Carmen cuestiona a su padre sobre la sopa de flor de calabaza porque debería ser más picante, a Martín no le gusta que su hija critique su comida.

Martín debe irse porque le piden que apoye en un banquete. Las hijas se quedan lavando trastes y discutiendo porque ya son adultas y siguen viviendo en la casa paterna, como se acostumbra en México pero no en Estados Unidos; se desahogan rompiendo platos y después ríen y cantan.

Martín llega al banquete y arregla un postre quemado de forma exitosa. Platica con Gómez sobre su familia, su trabajo, lo mucho que extraña a Ana, y le confiesa que ha perdido su capacidad de oler y saborear. 
Martín se encarga de las labores del hogar mientras sus hijas se alistan para ir a trabajar. Yolanda presenta a Hortensia, su mamá, con Martín y los deja solos para que se conozcan.

La vida de los protagonistas continúa, Leticia conoce a Orlando, un profesor de educación física y los alumnos de Lety le juegan una broma y le hacen creer que el profesor le manda cartas. Carmen se entrevista con un cliente de Barcelona y Maribel frecuenta a Andy. Hortensia busca a Martín tratando de que se interese en ella.

En la siguiente comida familiar Carmen anuncia que le han ofrecido un trabajo en Barcelona. En el restaurante, Gómez se desmaya y lo llevan al hospital, en donde lo visita Carmen, que le tiene mucho aprecio. Maribel lleva a Andy a cenar a su casa, él disfruta la comida. Maribel avisa a su familia que no estudiará por un tiempo y ante el enojo de su padre, decide irse a vivir con Andy. Lety sigue recibiendo cartas y se ilusiona. Cambia su peinado y su forma de vestir. Decidida, busca a Orlando y lo besa delante de alumnos y padres. Él está confundido y ella descubre la verdad. Lety se esconde en su salón de clases, muy avergonzada. Orlando la busca, la consuela y se besan.

En la siguiente cena familiar, en la que no está Maribel, Lety les habla de Orlando, que está afuera, esperando para entrar. Ya adentro, ambos explican que se fueron a Las Vegas y se casaron. Martín está sorprendido. Después de la cena, los recién casados se van juntos.

Maribel y Andy tienen problemas porque ella quiere mantenerlo todo en orden y a él no le gusta. Maribel lo deja y regresa a casa de su padre. Gómez regresa al restaurante pero muere casi de inmediato. El funeral es en casa de los Naranjo. Carmen está muy triste, discute con su papá por el viaje a Barcelona y se va de la casa. Carmen busca a Jeff y lo encuentra con otra mujer; le avisa que se irá a Barcelona. Andy busca a Maribel y se reconcilian, además de que estudiarán juntos.

La familia está junta de nuevo, con sus parejas y amigos. Martín tiene varios avisos que dar. Les dice que a pesar de que en esa casa han vivido momentos maravillosos, la ha vendido y compró una nueva. También les dice que se hizo una revisión médica completa y su salud es perfecta. Hay una última noticia, Hortensia cree que le pedirá matrimonio pero Martín confiesa estar enamorado de Yolanda, le pide que se casen y ella acepta.

Antes de irse a Barcelona, Carmen cocina pollo pibil para su papá y él considera que está muy picante, entonces se da cuenta que puede oler y sentir sabores de nuevo.
Carmen decide quedarse y hacer lo que siempre quiso, ser chef. En el restaurante lleva comida a la mesa en donde está reunida toda la familia. Yolanda aprovecha la ocasión para anunciar que el bebé que espera será una niña.

\section{Estoy poniendo la mesa}

La alimentación es una parcela importante de la antropología y condicionante importante de la evolución biológica del ser humano, se considera una necesidad básica para la supervivencia (Sánchez, 2017). Podría decirse que el siglo XX fue testigo de las revoluciones alimenticias más significativas desde que comenzó la agricultura hace 10.000 años, donde se han visto cambios radicales en la producción, proceso, distribución y consumo de alimentos. De esta forma la comida es un elemento clave para conocer la cultura, donde se desempeñan roles simbólicos importantes y ayuda a dar sentido a nuestras vidas. Forma parte de nuestra lengua, ritos y valores.

Al sentarnos a comer, no advertimos que en ese acto cotidiano se agazapa la historia entera de la humanidad. Comemos en grupo y abandonando la postura erguida; no comemos comida, comemos emblemas. El tacto es el contacto directo que media entre el olfato y el gusto: la mano lleva a la boca (Rossi, 2013).

En Tortilla Soup, la cena es sagrada. Es el momento donde Martín Naranjo convive con sus hijas, donde todos hablan de lo que les pasó en la semana, de los cambios que harán en sus vidas y de lo que es importante que se comparta.

Existen investigaciones que sugieren que las personas que comen regularmente con sus familias tienen un mejor reporte de salud que aquellas personas cuya frecuencia de comida familiar es baja (Schnettler et. al., 2013). La comida familiar es el momento en el que se reúnen todos para compartir; constituye una actividad central y un particular punto de encuentro en la vida diaria, vital para fortalecer la unidad y cohesión familiar (Beltrán y Cuadrado, 2014).

$\mathrm{Al}$ analizar los efectos de reunirse a comer en padres e hijos de familias de clase media en Estado Unidos, se observó que comer frecuentemente en familia disminuye el estrés de los padres generado por largas jornadas laborales y además mejora la relación entre éstos y sus hijos, por lo que concluyeron que la comida familiar es un ritual que tiene un rol protector frente al estrés de los padres, afectando positivamente el bienestar de los miembros del hogar. (Yoon et.al., 2015) 
Se puede demostrar la forma de actuar de Martín Naranjo en el postulado de Yoon et.al., pues al cocinar y poner la mesa, su estrés disminuía y se convertía en el mejor momento de la semana. Por el contrario, para sus hijas menores era un momento complicado. En cambio para Leticia, la hija mayor, que se volvió muy religiosa a partir del abandono de su esposo Carlos, era una buena experiencia.

Para Carmen la segunda hija, que estudió administración y es muy exitosa en su empleo, la cena familiar era muy estresante porque disfrutaba mucho cocinar y siempre encontraba peros en la forma en que Martín elaboraba los platillos debido a la pérdida de los sentidos del gusto y olfato. Maribel, la hija menor, acababa de terminar High School y estaba indecisa sobre ir a la universidad, lo cual le genera problemas con su padre y hermanas pues la consideran inmadura.

Conviene subrayar que comer en familia es un espacio de encuentro, pero no garantiza exclusivamente relaciones positivas, como se puede observar con la familia Naranjo. Existen investigaciones que afirman que puede ser muy abrumador el reunirse en una cena familiar, pues se puede convertir en un espacio de conflicto y tensión (Neumark-Sztainer et.al., 2000). Este es el caso de las hijas Naranjo, ya que para ellas esta reunión es como una restricción a su autonomía. Y desde la perspectiva de Martín en ocasiones se convierte en un campo de batalla.

Por otro lado, la comida familiar tiene un rol fundamental en cuanto a la socialización de las personas y la transmisión de valores. Algunos investigadores sostienen que la cena en familia actúa como una plataforma que facilita que se adquieran e interioricen normas y valores (Paugh e Izquierdo, 2009). Se debe agregar que es un espacio cultural en el cual se negocian y construyen relaciones interpersonales por medio del relato de las experiencias, ideas y creencias cotidianas (Ochs y Shohet, 2006; Sterpoli, 2009).

Hay que mencionar que cuando Martín se tuvo que ir a trabajar, las chicas se quedaban solas para lavar la loza y acomodar todo en su lugar, cantaban y bailaban la canción Quizás, quizás, quizás... (1947) de Joe Davis y Osvaldo Farrés, en inglés y español, apuntando a sus dos raíces, la estadounidense y la mexicana.

Este momento mezcla comida, baile y música, que dan por resultado tradiciones que tienen las familias al reunirse a degustar los alimentos.

\section{Las tradiciones México - Estados Unidos}

La cocina es uno de los aspectos de la vida cotidiana que refleja el desarrollo de un pueblo; comprende el aprovechamiento preciso de las posibilidades nutritivas y gustativas de todos los productos que pueden servir como alimento a una población (Torre, 2012). No es únicamente su poder nutritivo lo que convierte a un producto vegetal o animal en un alimento, sino también el uso que da a los productos cada grupo social. Cada cultura tiene su propia definición de lo que es comestible y de lo que no lo es (Flandrin 1987).

La cocina juega un lugar esencial en la cultura, ya que a través de esta se representan sabores, gustos, costumbres, tradiciones, valores, etc., los cuales se ven inmiscuidos al momento de preparar un alimento, en los utensilios de cocina con sus significados y funciones de acuerdo al entorno, en la organización y motivo para su elaboración, y en las distintas costumbres y tradiciones que lo acompañan. (Ochoa y Santamaría, 2010, s/d).

En la cinta, Martín sale al jardín de su casa a cortar legumbres que cultiva en un pequeño huerto para poder preparar la cena del domingo. Asa chiles en un asador de carbón. Corta nopales de una penca, les quita las espinas y también los pone a asar. Limpia y corta hojas de plátano para untar achiote ${ }^{1}$ en unos pescados que asa al carbón. Lava y desinfecta flor de calabaza que sofríe con ajo, cebolla y chiles jalapeños desvenados para hacer una sopa. En un molcajete pone cebolla, tomates y chile para hacer salsa. De postre prepara dulce de calabaza y nieve que pone en un bote de madera rodeado de mucho hielo y sal. Los platos son de barro del estado de Oaxaca y los vasos de vidrio soplado con un entintado color verde en el borde, muy del estado de Puebla.

Las tres hijas Naranjo viven en la casa de su padre. Hablan spanglish ${ }^{2}$, debido a que han creado un conjunto de características únicas ligadas a la manera en la que se organizan en su entorno cultural. Cocinar y comer expresan formas y estilos de vida, las cuales se van adquiriendo durante su interacción con los demás.

Los Naranjo conviven con su vecina Yolanda que tiene una hija llamada April y su mamá Hortensia, que acaba de llegar para ayudarla con su divorcio. Yolanda no tiene tiempo para prepararle un buen desayuno a April, así que Martín hace un plan con la niña para hacer su almuerzo y a partir de la comida que empieza a llevar a la escuela se vuelve muy famosa e inicia un lazo paternal con el chef Naranjo.

En los elementos de la cultura se encuentran formas llamadas objetivas e interiorizadas, la primera tiene que 
ver con todos los objetos cotidianos, religiosos y rituales, los cuales tienen la característica de ser observados con mucha facilidad, la segunda forma es en la que la persona crea conceptos en su mente y está relacionada con las formas simbólicas y estructuras mentales (Bourdieu, 1998). De esta forma la cultura de una sociedad muestran la trascendencia y la forma de vivir de una comunidad, por lo tanto, cocinar es una expresión cultural. Por un lado, están los platillos y bebidas que son formas culturales objetivas y la forma interiorizada que es la forma en cómo son preparados y degustados los alimentos.

Sin duda, comer, alimentarse y encontrar en la mesa las más profundas referencias propias de un pueblo y su sociedad, son parte de los derechos fundamentales y más deleitables de los hombres y las mujeres (Lody, 2002).

Por otro lado, existen varias líneas de progreso que han contribuido a que una proporción creciente de hombres aprovechen tradiciones culinarias cada vez más elaboradas (Cordón, 1980). Es aquí donde podemos hacer una referencia al trabajo que realizaba Martín, pues no solo se llevó la tradición de la comida mexicana a Estados Unidos, sino que hizo una mezcla con las dos cocinas y las hizo suyas. Al mismo tiempo, Carmen fusiona su cocina con Francia, México, Sudamérica y el Caribe, y eleva la gastronomía a un nivel internacional.

El disfrute gastronómico se despega del placer puramente animal por dos condiciones, la primera es el afinamiento, la educación del paladar, su ajuste a lo bien cocinado conforme a tradición, que permite distinguirlo del plato burdo o mal hecho; la segunda es la percepción y el aprecio de un matiz de sabor nuevo que armonice sabiamente el respeto a la tradición con la innovación creadora (Cordón, 1980). Tanto Martín como Carmen transforman la gastronomía mexicana y la fusionan con otras raíces para darle su toque personal sin olvidar de dónde vienen.

\section{La ética y los alimentos}

La alimentación no cubre solamente una necesidad básica del ser humano, implica aspectos más profundos y complejos como las relaciones interpersonales, el formar parte de una sociedad, el establecimiento de tradiciones familiares y culturales o el sentido de pertenencia. Es aquí donde la ética tiene un papel preponderante pues cocinar, compartir los alimentos y establecer lazos y rutinas que se repiten de generación en generación requiere de parámetros para que los rituales gastronómicos sean aceptados, aprehendidos y transmitidos. Este intercambio se logra gracias a la necesidad que tenemos de socializar y la capacidad de hacerlo a través del lenguaje.

El lenguaje no tiene sentido si no es para esta apertura a los demás. Esto se comprueba porque la falta de diálogo es lo que motiva casi todas las discordias y lo que arruina las comunidades humanas (matrimonios, familia, empresas, instituciones políticas, etc.) Sin comunicación no hay verdadera vida social, a lo sumo apariencia de equilibrio, pero falta el terreno común sobre el cual poder construir. (Yepes y Aranguren, 2003, s/d).

En Tortilla Soup el lenguaje es clave en la comunicación familiar desde dos perspectivas: la forma y el fondo.

En relación con la forma, el lenguaje utilizado por las protagonistas es híbrido. Las hijas de Martín mezclan español e inglés para comunicarse en casa, no porque no puedan hablar por completo en español, su lengua materna, o porque no dominen el inglés, sino porque a raíz de la mezcla entre ambas culturas, se ha creado una forma de comunicación que combina ambos idiomas. El que sus hijas hablen spanglish molesta a Martín y les pide que elijan una lengua u otra pero que no las mezclen. Esto podría deberse a la necesidad de respetar tradiciones y mantenerlas vivas, para no perder identidad.

En cuanto al fondo, el lenguaje se convierte también en herramienta indispensable de integración. Las reuniones dominicales de los Naranjo buscan que además de la convivencia natural que se da al comer con los seres queridos, se abran espacios para compartir con la familia cuestiones de suma importancia que cambiarán la rutina tanto del que da la noticia como de todos los que le rodean.

A lo largo del film se van tomando decisiones que tienen que ver no solo con la familia, sino con los amigos y conocidos de los Naranjo. Cuando Gómez llama a Martín porque sus ayudantes echaron a perder el postre para un importante miembro del gobierno de Estados Unidos, le pide despedir a todos. Naranjo mira las caras preocupadas de sus trabajadores y le dice a Gómez que no lo volverán a hacer y que no despedirán a nadie. Hacen capirotada ${ }^{3}$ pero le ponen un nombre francés para que suene más fino y así resuelven el problema.

Martín tomó una decisión que pudo hacer que la vida de sus empleados cambiara radicalmente y que sus familias ya no tuvieran dinero para sobrevivir. Los valores de Martín hicieron que no despidiera a nadie, además evitó que su reputación como chef se viera afectada.

Existen dos ideales éticos fundamentales: el kyosei y la dignidad humna. El primero significa vivir y trabajar juntos para el bien común, permitiendo que la cooperación y la prosperidad mutuas coexistan con una compe- 
tencia justa y saludable. Por dignidad humana se entiende el supremo valor de la persona como fin, y no como medio, para la consecución de los objetivos de otros, incluso de la mayoría (Chomali y Majluf, 2007).

El hombre, considerado en toda su riqueza y en todo su valor, es el fundamento de la ética. Para juzgar si sus actos son de bondad o maldad, se debe partir de su impacto sobre el hombre, su bienestar, felicidad; su mejoramiento como persona. A la persona le compete la tarea de desarrollar todas sus capacidades en los ámbitos en los que se desarrolla su vida para llegar a su plenitud como ser humano. Toda persona tiene derecho de ser el autor principal de su progreso, y resulta éticamente inaceptable realizar acciones que vulneren la libertad de las personas o que se manipule su conciencia.

En la imagen 1 se ejemplifica la construcción del carácter y la toma de decisiones.

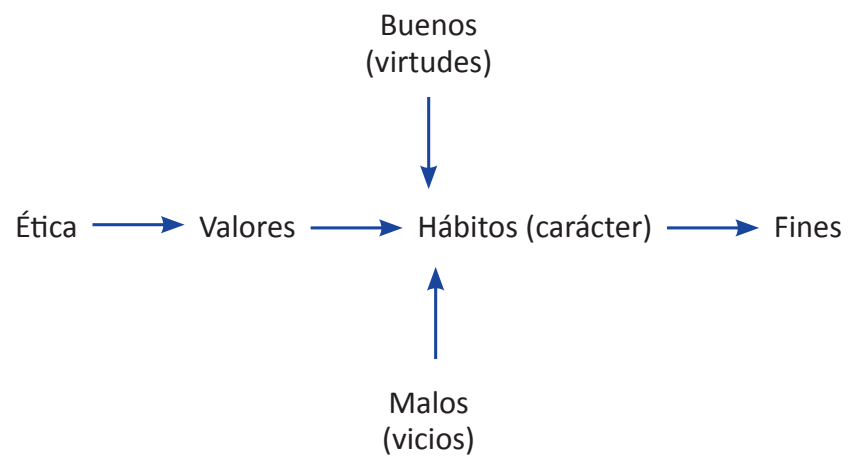

Imagen 1 .

La construcción del carácter o cómo aprender a tomar decisiones. Fuente: Elaboración propia.

En este cuadro se muestra la relación entre el actuar humano y sus resultados. Tanto los buenos hábitos como los vicios forman el carácter de las personas y determinan las decisiones que toman. Aristoteles sentó las bases del actuar del hombre en relación con su plena realización; el hombre requiere de la ética para centrar su quehacer en el bien de la persona misma.

[...] un conjunto complejo de nuestras tendencias e inclinaciones innatas, herencia heredada en gran medida por nuestros antepasados, constituye nuestro "carácter psicológico", que los expertos en el campo han clasificado y subdividido en numerosos "tipos”. Es lo que comúnmente llamamos “el temperamento individual”. (Freire, 1997, p. 410)

Según Aristóteles, el deseo es el principio de la acción, a este le sigue la deliberación que lleva a su vez, a la elección. Una vez hecha esta elección, sigue como consecuencia el acto moral. Por ello el hombre debe equilibrar el deseo y la razón y ejercitar la prudencia, que es un hábito práctico y verdadero acompañado de razón, con relación a los bienes humanos (Vallejo, 2006).

Es por esto que las personas tienen, a lo largo de sus vidas, dilemas éticos que los enfrentan a decisiones difíciles que requieren de atención, discernimiento y análisis. Estas decisiones producen un impacto sobre otras personas y sobre la sociedad, por lo que se debe actuar con justicia, verdad y prudencia. Actuar es solo un aspecto de la vida buena, ya que vivir bien supone sobre desarrollar un determinado carácter, una especial manera de ser (Aristóteles, 1983).

$\mathrm{Si}$ alguien es modelo de carácter y comportamiento a seguir para sus familias o empleados es Martín Naranjo, pues marca una pauta de comportamiento. En casa de los Naranjo se siguen modelos como el ritual de bendecir los alimentos o de no faltar a la cena familiar de los domingos que llaman "hora de convivencia" y es inamovible.

Surge, desde luego, un actuar individual en la toma de decisiones de cada hija. Martín no comparte sus puntos de vista y necesita tiempo para asimilar los cambios que van surgiendo pero termina confiando en que ellas sabrán elegir lo que cada una considere más conveniente.

La virtud moral, en efecto, se relacionará con el placer y el dolor en la medida en que hacemos el bien a causa del placer que nos produce y nos apartamos del mal igualmente porque nos produce dolor. Pero, dado que no siempre el placer y el dolor coindiden con el bien y el mal morales, la tendencia a buscar el placer y evitar el dolor deberá corregirse, mediante la educación, a fin de que aprendamos a colocar el placer y el dolor en aquello que debe producirlo. El hombre bueno es el que acierta a designar lo bello, lo conveniente y lo agradable, y sus contrarios, lo vergonzoso, lo perjudicial y lo penoso. (Aristóteles, 1990, s/d)

Otro de los elementos mostrado en la película que es importante destacar es el de los migrantes, pues como no entran al país legalmente son víctimas de malos tratos, jornadas de trabajo muy largas y los pagos que reciben son muy bajos. Martín empezó así y pudo ser un jefe injusto, exigente y poco flexible cuando logró tener su propio restaurante, pero no lo hizo y da a sus trabajadores, en su mayoría inmigrantes, un trato justo y humano.

El conocimiento de la propia identidad, la conciencia de uno mismo, sólo se alcanza mediante la intersubjetividad. Este proceso de intercambio constituye la formación de la personalidad humana. En él se modula el propio carácter, se asimila el idioma, las costumbres y las instituciones de la colectividad en que se nace, se incorporan sus valores comunes, sus pautas, etc. (Yepes y Aranguren, 2003). 
Estados Unidos ha dependido de la labor de las personas desplazadas, sin poder político, sin protecciones de trabajo, sin derecho a votar y sin contar con todos los derechos que tienen los ciudadanos. Además, han sido separados de sus seres queridos por la militarización de la frontera de Estados Unidos con México. Los trabajadores aún hacen frente a estos obstáculos hoy en día.

Finalmente, es importante hablar de la relevancia del protagonista, pues constituye el punto de unión entre el resto de los personajes. Martín Naranjo dedica tiempo a la reflexión, analiza su forma de actuar y de reaccionar, escucha el punto de vista de los demás, reconoce sus errores y cambia lo que sea necesario para que vuelva a haber armonía.

El conocimiento de la propia identidad, la conciencia de uno mismo, sólo se alcanza mediante la intersubjetividad. Este proceso de intercambio constituye la formación de la personalidad humana. En él se modula el propio carácter, se asimila el idioma, las costumbres y las instituciones de la colectividad en que se nace, se incorporan sus valores comunes, sus pautas, etc. (Yepes y Aranguren, 2003, p. 68).

\section{A manera de cierre}

El film Tortilla Soup es un reflejo de muchas familias que tienen que emigrar a otro país y se llevan su cultura y tradiciones al nuevo lugar a donde llegan. Desmenuza la cultura gastronómica que simboliza una suerte de influencia que combina energía con imaginación, sacrificio con jovialidad, supervivencia con esperanza, serenidad con ingenio, y que encuentra una mayor definición en la búsqueda de su perfeccionamiento y excelencia. Es el reflejo perfecto de que alimentarse no solo cubre necesidades biológicas, sino que combina perfectamente con aspectos culturales, familia, migración y ética.

Los hispanos son un grupo que crece de manera acelerada en Estados Unidos y aporta hábitos alimenticios basados en la cultura a medida que crecen. Incluso aquellos que nacieron en EE.UU. continúan enfatizando la importancia de la cocina tradicional latinoamericana cuando preparan comidas familiares.

Las crecientes poblaciones de migrantes han introducido nuevos sabores en la dieta de los estadounidenses y muchos de ellos son pilares en la gastronomía, de ahí la importancia del impacto de las culturas, pues genera nuevos mercados, campos laborales e ingresos.
La alimentación en la mayor parte de los países occidentales está cambiando de necesidad a pasatiempo. La comida se ha vuelto rápida y cómoda, pero paradójicamente hay más afición a la cocina, y de aquí la importancia de que se siga incorporando en los medios de comunicación, pues es el puente para compartir experiencias, significados y emociones.

Como respuesta al objetivo de esta investigación tanto en los migrantes que viven en Estados Unidos, como en su representación en medios audiovisuales, se puede apreciar que hay tradiciones que no se debilitan al emigrar a otro país, siempre y cuando persista un interés por mantener vivas las raíces del lugar de origen. Sin embargo, sí surge la combinación cultural de dos mundos que se fusionan creando nuevos lenguajes y costumbres.

Las hijas Naranjo son la segunda generación de mexicanos viviendo en Estados Unidos, y se puede apreciar que tienen mejor calidad de vida que sus padres cuando llegaron. Ellas son mexico-americanas y por lo tanto, tuvieron la posibilidad de ir a la universidad (en el caso de las dos mayores) y la más joven accede a estudios superiores. Esto es a lo que pueden aspirar las segundas y terceras generaciones de inmigrantes, a tener una mejor calidad de vida sin perder la cultura de sus padres y formar una nueva, que surge de la combinación de los estilos de vida que van adquiriendo a lo largo de sus vidas.

La comida en este film se convierte en pieza clave tanto en su narrativa como en su representación de la realidad, pues como en las vidas de los mexicanos los sabores, colores y olores son parte de las tradiciones, costumbres que también involucran aspectos como la integración familiar, el respeto y el actuar cotidiano para alcanzar el bien común de las familias.

Los Naranjo toman decisiones que afectan la vida de cada uno de los integrantes, pero su sentido de pertenencia y los valores aprendidos de sus padres les lleva a discernir sobre qué es lo correcto o no, pues fueron educadas a decir siempre la verdad, sin esconder ni distorsionar la información.

Por todo lo anterior y a pesar de que no puede hablarse de la totalidad de los inmigrantes, la comida hace que familias de mexicanos en Estados Unidos mezclen sus raíces con nuevas tradiciones y sientan el orgullo de sus antepasados al tiempo que buscan una mejor calidad de vida, lo que se verá reflejado en una mejor economía, una mejor educación y mejores formas de relacionarse como seres humanos. 


\section{Referencias}

Alba, R. y Nee, V. (2003). Remaking the American Mainstream. Assimilation and Contemporary Immigration. Massachusetts, Estados Unidos: Harvard University Press.

Aristóteles. (1983). Ética nicomáquea [traducción de Antonio Gómez Robledo]. México: Universidad Nacional Autónoma de México. $2^{a}$ edición.

Aristóteles. (1990). Retórica [traducción de Quintín Racionero]. Madrid, España: Gredos.

Bard Manulis, J. (productor) y Ripoll, M. (directora). (2001). Tortilla Soup [cinta cinematográfica]. Estados Unidos: Samuel Goldwyn Films.

Beltrán, B., y Cuadrado, C. (2014). Comer en familia: hacer de la rutina salud. Papel de las comidas en familia en la calidad de la dieta y el control del peso corporal. Departamento de Nutrición y Bromatología. Facultad de Farmacia. Universidad Complutense de Madrid. The Family Watch.

Bourdieu, P. (1998). Creencia artística y bienes simbólicos. España: Deva’s.

Chomali, F., y Majluf, N. (2007). Ética y responsabilidad social en la empresa. Chile: Mercurio Aguilar.

Cordón, F. (1980). Cocinar hizo al hombre. Barcelona: Tusquets editores.

Cruz Piñeiro, R., y Acosta Díaz, F. (Coord) (2015). Migración interna en México. Tendencias recientes en la Movilidad interestatal. México: El Colegio de la Frontera Norte.

Flandrín, J.L. (1987). Historia de la alimentación: por una ampliación de las perspectivas. Manuscrits: Revista d’història moderna, (6), 7-30.

Freire, P. (1997). Sobre la necesidad de prestar atención, en la escuela, a la educación del carácter, así como al cultivo de la personalidad del niño. Conferencia Nacional de Educación: Curitiba, 1927. Brasilia: MEC; SEDIAE; Inep Ipardes. 410-415.

Hsu, K., Hsu, L.K., Jiang, F. (productores) y Lee, A. (director) (1994). Comer, beber, amar [cinta cinematográfica]. Taiwán: The Samuel Goldwyn Company.

Lody, R. (2002). Comida y patrimonio. Caju: fruto emblema de la identidad brasileña. Patrimonio Cultura y Turismo. Cuadernos 9. Patrimonio cultural oral e inmaterial. La discusión abierta. Antología de textos. México: Consejo Nacional para la Cultura y la Artes.

Neumark-Sztainer, D., Story, M., Ackard, D., Moe, J., y Perry, C. (2000). The Family Meal: Views of Adolescents. Journal of Nutrition Education and Behavior, 32, (6), 329-334.

Noe-Bustamante, L., Alberti, D., y Rodriguez-Gitler, A. (2019). Hispanic origin profiles, 2017. Pew Research Center. Hispanic Trends. Recuperado de https://www.pewresearch.org/hispanic/interactives/hispanic-origin-profiles/

Ochoa, K., y Santamaría, A. (2010). Cultura e identidad culinaria. Arenas Revista sinaloense de ciencias sociales, (25), 78-91.

Ochs, E. y Shohet, M. (2006). The Cultural Structuring of Mealtime Socialization. New Directions for Child and Adolescent Development, 2006(11), 35-49.

Paugh, A, e Izquierdo, C. (2009). Why is This a Battle Every Night?: Negotiating Food and Eating in American Dinnertime Interaction. Journal of Linguistic Antropology, 19(2), 185-204.

Pérez-Soria, J. (2017). Migrantes mexicanos en los Estados Unidos: Una revisión de la literatura sobre integración, segregación y discriminación. Estudios fronterizos, 18(37), 1-17.

Portes, A. y Zhou, M. (1993). The New Second Generation: Segmented Assimilation and its Variants. Annals of the American Academy of Political and Social Science, (530), 74-96.

Radford, J., y Noe-Bustamante, L. (2019). Facts on U.S. Immigrants, 2017. Statistical portrait of the foreign-born population in the United States. Pew Research Center. Hispanic Trends. Recuperado de https://www.pewresearch.org/hispanic/2019/06/03/facts-onu-s-immigrants-current-data/

Rossi, L. (2013). Historia del comer. Lazo social y tradición cultural. Intersecciones PSI. Revista electrónica de la Facultad de Psicología-UBA, (7), 16-18.

Sánchez, A. (2017). Antropología de la alimentación. Unidad de nutrición. Hospital general universitario Reina Sofía de Murcia. España.

Schnettler, B., Denegri, M., Miranda, H., Sepúlveda, J., Orellana, L., Paiva, G., Grunert, K. (2013). Hábitos alimenticios y bienestar subjetivo en estudiantes universitarios del sur de Chile. Nutrición hospitalaria, 28(6), 2221-2228.

Sterponi, L. (2009). Accountability in Family Discourse: Socialization into norms and standards and negotiation of responsibility in Italian dinner conversations. Childhood: A Global Journal of Child Research, 16(4), 441-459.

Torre, M.C. (2012). La ciencia de los alimentos. Lo que hay detrás de las recetas de cocina. México: Trillas. 
Vallejo, X. (2006). Carácter, razón y pasión en la ética de Aristóteles. Criterio Jurídico. Santiago de Cali, 6, $327-352$.

Yepes Stork, R., y Aranguren Echevarría, J. (2003). Fundamentos de la Antropología. Un ideal de la excelencia bumana. Navarra: EUNSA.

Yoon, Y., Newkirk, K., y Perry-Jenkins, M. (2015). Parenting Stress, Dinnertime Rituals, and Child Well-being in Working-Class Families. Fam Relat, 64(1), 93-107.

1 Es una planta arborescente que crece en México, América Central y en las zonas cálidas de los Andes que hacen límite con la Amazonia.

2 Es un término popular con el que se denomina a ciertas variedades del español hablado en Estados Unidos, en contextos en el que el español e inglés están en contacto prolongado debido a la presencia de personas bilingües.

3 Es un postre popular mexicano hecho con rodajas de pan fritas, bañadas con jarabe de piloncillo y adornadas con almendras, cacahuates, uvas pasas y queso blanco salado. 